Sammlung Metzler Band 275 


\author{
Andreas Schrade
}

\title{
Anna Seghers
}

Verlag J.B. Metzler

Stuttgart - Weimar 
Die Deutsche Bibliothek - CIP-Einheitsaufnahme

\author{
Schrade, Andreas: \\ Anna Seghers / Andreas Schrade. \\ - Stuttgart ; Weimar : \\ Metzler, 1993 \\ (Sammlung Metzler ; Bd. 275) \\ ISBN 978-3-476-10275-1 \\ NE: GT
}

\author{
ISSN 05583667 \\ ISBN 978-3-476-10275-1 \\ ISBN 978-3-476-03973-6 (eBook) \\ DOI 10.1007/978-3-476-03973-6
}

SM 275

Dieses Werk einschließlich aller seiner Teile ist urheberrechtlich geschützt. Jede Verwertung außerhalb der engen Grenzen des Urheberrechtsgesetzes ist ohne Zustimmung des Verlages unzulässig und strafbar. Das gilt insbesondere für Vervielfältigungen, Übersetzungen,

Mikroverfilmungen und die Einspeicherung und Verarbeitung in elektronischen Systemen.

(C) 1993 Springer-Verlag GmbH Deutschland

Ursprünglich erschienen bei J. B. Metzlersche Verlagsbuchhandlung und Carl Ernst Poeschel Verlag GmbH in Stuttgart 1993 


\section{Inhaltsverzeichnis}

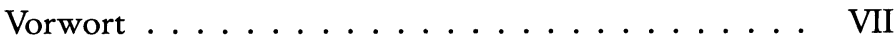

I. Die frühen Erzählungen - Krisenstimmung und Aufbruch . . . . . . . . . . . . 1

1. "Die Toten der Insel Djal. Eine Sage aus dem

Holländischen. Nacherzählt von Antje Seghers» . . . 5

2."Grubetsch" ............... 6

3. "Aufstand der Fischer von St. Barbara" . . . . . . 11

4. »Auf dem Wege zur amerikanischen Botschaft und andere Erzählungen «. Erster Erzählungsband . . . . 15

II. Die frühen Erzählungen im Spiegel der kommunistischen Literaturkritik

III. Ästhetisches Selbstverständnis . . . . . . . . . . 27

IV. "Die Gefährten« - Roman der kommunistischen Weltrevolution ............... 36

V. Exil ................... 40

1. "Der Kopflohn" - Das Fußfassen des Faschismus im deutschen Volk . . . . . . . . . . . 40

2. "Der Weg durch den Februar « - Beispiel eines Aufstands gegen eine faschistische Regierung . . . 43

3. "Die Rettung" . . . . . . . . . . . . . . . 47

3.1. Romantypus Seghers . . . . . . . . . . . 47

3.2. Darstellung der Krise . . . . . . . . . . . . . 49

4. Erzählungen und Publizistik im französischen Exil . $\quad 53$

5. "Das siebte Kreuz" - Leben unter der faschistischen Diktatur . . . . . . . . . . . 61

6. "Transit«-Beschreibung des entwurzelten Lebens . 66

7. Nationale Besinnung und ästhetisches Programm. Überlegungen im mexikanischen Exil . . . . . 70

VI. "Die Toten bleiben jung « - Panorama der deutschen Geschichte 1918-1945 _ . . . . . 78 
VII. Erste Erzählungen der Nachkriegszeit . . . . . . .

VIII. Der Schriftsteller als Propagandist des sozialistischen Aufbaus. Standortbestimmung nach der Rückkehr aus dem Exil . . . . . . . . . . .

IX. Wege zur Darstellung des Neuen . . . . . . . . . 103

1. Erste Annäherungen - "Friedensgeschichten " . . . . 103

2. Der revolutionäre Umbruch als individuelles Identitätsproblem - "Der Mann und sein Name« . . . . . 108

X. "Die Entscheidung - Epochenumbruch als einheitlicher Prozeß . . . . . . . . . . .

XI. "Das Vertrauen« - Die neue Gesellschaft als Einheit der Widersprüche . . . . . . . . . 118

1. Gesellschaftliche Entwicklung und Figurenkonstellation . . . . . . . . . . . . . 118

2. Der 17. Juni - Bewährung, nicht Entscheidung . . . . 123

XII. "Das wirkliche Blau« - Der Mensch außerhalb der modernen geschichtlichen Welt . . . . . . 126

1. Zur Vorgeschichte - "Das Licht auf dem Galgen " und "Die Kraft der Schwachen" . . . . . . . . . 126

2. Die Fragwürdigkeit der individuellen Identität "Das wirkliche Blau« . . . . . . . . . .

XIII. »Überfahrt. Eine Liebesgeschichte« - Die individuelle Beziehung als Grundlage der Identität . .

XIV. "Sonderbare Begegnungen « und "Steinzeit / Wiederbegegnung - Fragen der Kunst und Orientierung auf das Individuum . . . . . . .

XV. »Drei Frauen aus Haiti« - Letzte Fragen einer Revolutionärin . . . . . . . . . . . . . . 152

Zeittafel . . . . . . . . . . . . . . . 156

Literaturverzeichnis . . . . . . . . . . . . . . . . 159

Namenregister . . . . . . . . . . . . . . . 166

Angaben zum Autor . . . . . . . . . . . . . . . . 169 


\section{Vorwort}

Anna Seghers war eine politisch sehr bewußte und engagierte Schriftstellerin: Kommunistin, seit 1928 Mitglied der KPD, seit ihrer Rückkehr aus dem Exil in der SED. So ist es nicht verwunderlich, daß die Wirkungsgeschichte ihres umfangreichen erzählerischen Werks zum größten Teil eine Auseinandersetzung mit der kommunistischen Weltanschauung und der entsprechenden politischen Haltung der Autorin ist. Die Stimmen pro und kontra Anna Seghers sind folglich ziemlich leicht auseinanderzuhalten. Selbst dort, wo scheinbar unpolitische Seghers-Philologie betrieben wird, schimmert das Ja oder Nein zur politischen Haltung der Verfasserin noch durch.

Diese Wirkungsgeschichte begann am Ende der Weimarer Republik. Anna Seghers hatte 1928 den hochangesehenen Kleist-Preis erhalten, damals eine denkbar günstige Voraussetzung für eine literarische Laufbahn. Schon die ersten Reaktionen auf das Debüt "Aufstand der Fischer von St. Barbara « (1928) und auf die Preisverleihung zeigen unverkennbar: Wer für Anna Seghers war, der unterstützte auch ihr soziales Engagement (von direkter politischer Parteinahme kann in dieser ganz frühen Phase noch keine Rede sein). Wer gegen die junge Autorin war, der stand auch ihrem Engagement fremd gegenüber.

Im Exil finden dann die literarischen Debatten nur noch in den festen Zirkeln der einzelnen Exilgruppen statt. Anna Seghers gelingt unter diesen Bedingungen ein großer internationaler Erfolg, was nur sehr wenige deutsche Schriftsteller in der Emigration erreichten. "Das siebte Kreuz" erscheint 1942 in den USA, erobert dort sofort den ersten Platz auf einer Bestsellerliste (vor Franz Werfels »Das Lied von Bernadette«) und wird mit ebenso großem Erfolg in Hollywood verfilmt (Regie: Fred Zinnemann, Spencer Tracy als Georg Heisler).

Als Anna Seghers 1947 nach Deutschland zurückkehrt, ist sie vor allem die berühmte Autorin des »Romans aus Hitlerdeutschland «, "Das siebte Kreuz«. Sie erhält 1948 in Darmstadt den Büchner-Preis. Der Verleger Ledig-Rowohlt nimmt den Roman in seine neu geschaffene Taschenbuchreihe Rowohlts Rotationsromane auf, die Auflagenhöhen von bisher 
unbekanntem Ausmaß hat. Der Weg scheint bereitet für die neue Karriere einer deutschen Schriftstellerin von Weltruhm.

Dann kommt die Teilung Deutschlands und mit ihr zerfällt die Seghers-Rezeption in zwei Teile (ganz entgegen den Wünschen und Vorstellungen der Autorin). Die Rezeption im Westen, längst nicht so umfangreich wie die im Osten, ist weitestgehend aufgearbeitet (Buthge, 1982; Degemann, 1985). Sie wird zu einem erheblichen Teil - jedenfalls was die 50er und 60er Jahre angeht - von Ignoranz und Feindseligkeit bestimmt. Die Rezeption im Osten ist noch nicht systematisch erfaßt worden, dafür ist sie sehr eindeutig und folglich ziemlich leicht zu klassifizieren. Anna Seghers' Werk ist hier vor allem als Bestätigung (aktuell und geschichtlich) bzw. Unterstützung des politischen Programms der SED gedeutet worden. Nach einigen anfänglichen Reibereien wegen ihres Romans »Die Toten bleiben jung " von 1949 wurde Anna Seghers rasch in die Reihe der repräsentativen sozialistischen Schriftsteller gestellt: repräsentativ wiederum im aktuellen und im geschichtlichen Sinn. Anna Seghers hat diese Rolle keineswegs angestrebt (wie das wohl zeitweilig bei J.R. Becher der Fall gewesen ist). Sie stand öffentlich ohne Wenn und Aber für die sozialen, ökonomischen und politischen Veränderungen im Osten ein und hat sich auch nicht vor der Übernahme entsprechender Verantwortung gescheut. Sie bekleidete hohe Ämter: Präsidentin des Schriftstellerverbandes seit seiner Gründung, Vizepräsidentin des Kulturbundes. Politischen Ämtern innerhalb der Ministerien oder der Partei ist sie allerdings stets aus dem Weg gegangen. Man kann im übrigen vermuten, daß sie als sog. Westemigrantin dafür auch kaum in Frage kam.

Wegen dieser Rolle, die sie in der DDR spielte - die ihr vielmehr angetragen wurde und die sie unter dauernden Versuchen, sie nach dem eigenen, nicht dem vorgegebenen Muster zu gestalten, annahm - ist sie 1989/90 heftig attackiert worden. Durch die Veröffentlichung der Erinnerungen ihres ehemaligen Verlegers Walter Janka, »Schwierigkeiten mit der Wahrheit« (1989, inzwischen erheblich erweitert unter dem Titel "Spuren eines Lebens «) ist sie als Mitläuferin, wenn nicht gar als Feigling verschrieen worden. Janka wurde in den 50er Jahren als angeblicher Verräter und Aufwiegler angeklagt und verurteilt. Während der Gerichtsverhandlung schwieg Anna Seghers - was Janka ihr vorwirft. Inzwischen ist bekannt geworden, daß sie damals zu Walter Ulbricht gegangen war, um ihn zur Niederschlagung des Prozesses zu bewegen. (Vgl. dazu das Gespräch 
mit Anna Seghers' Tochter Ruth Radvanyi in: Sonntag 19/1990, S. 7.)

Die Wirkungsgeschichte des Seghersschen Werkes ist also ein getreues Spiegelbild der zerrissenen Welt, in der die Autorin lebte. Die geschichtlichen Veränderungen der letzten Jahre bringen nun eine ganz und gar veränderte Situation für die Einschätzung von Anna Seghers mit sich. Das politische Regime, das sie unterstützte, ist an seiner ökonomischen Unzulänglichkeit und diktatorischen Herrschaftsform zugrunde gegangen. Heißt das aber, Anna Seghers war eine Autorin (wie einige bereits gefolgert haben), die sich bewußt in den Dienst einer Diktatur stellte? Könne also ihr Werk bis auf wenige Ausnahmen, die von solch einer Haltung frei seien (»Das siebte Kreuz«, »Transit«, "Ausflug der toten Mädchen«), für die Zukunft kaum noch Bedeutung beanspruchen? Wer so urteilt, sieht vollkommen vorbei an der 'Welt ' dieses erzählerischen Werks - einer 'Welt', die sich nicht nach diesem oder jenem Ideologem bemessen läßt. Sind z.B. die Erzählungen der 70er Jahre das Produkt einer politischen Schriftstellerin, die nichts anderes im Sinn hatte, als die DDR und die entsprechende politische Ideologie zu bekräftigen? Das Gegenteil ist doch hier der Fall. Diese Texte zeigen sehr deutlich: Anna Seghers ist in der letzten Phase ihres Lebens sehr unsicher geworden hinsichtlich der geschichtlichen Wirklichkeit, des geschichtlichen Wandels und auch des Menschen überhaupt. Von Bestätigung eines vorgegebenen Wegs, von der Überzeugung, die proletarischen Revolutionen dieses Jahrhunderts seien der Eintritt ins Reich der Freiheit, kann in diesen späten Werken keine Rede mehr sein. (Das gilt übrigens bereits für "Das wirkliche Blau " von 1967 und in gewisser Weise auch für den Roman »Das Vertrauen« von 1968.)

Für große Teile des Gesamtwerks gilt: Man muß die 'Welt darin hinter der Verstrickung in die jeweilige Realität und Ideologie aufspüren. Will man die Romane und Erzählungen mit in eine Zukunft nehmen, die ganz andere Wege gehen wird als sie Anna Seghers im Sinn hatte, dann muß man vor allem die utopischen (humanistischen) Momente in ihnen wahrnehmen die 'Brüche' nicht als Manko registrieren, sondern als den Ort, der für unser Selbstverständnis in der Gegenwart von Bedeutung sein kann.

Dieser Gedanke liegt der folgenden Einführung in Leben und Werk von Anna Seghers zugrunde. Er bestimmt die Richtung, die bei der Analyse der Werke eingeschlagen wird. Anna Seghers Biographie ist bisher nur in groben Zügen bekannt. Anna 
Seghers' Devise war stets: Was zählt, ist das Werk, nicht das Leben der Autorin. Trotzdem würde eine gründliche Erhellung der Biographie dieser Schriftstellerin ganz sicher ein wertvoller Beitrag zum Verständnis der kommunistischen Bewegung in diesem Jahrhundert sein. Denn hier harrt noch immer vieles der Aufklärung. Wie sah die tatsächliche Beziehung dieser Schriftstellerin zu ihrer Partei aus? Die öffentlichen Bekundungen sind ja nur ein Teil der Wahrheit. Und wie verhielt sich die Partei zu ihr? In der Weimarer Republik? In der Emigration? Wie hat Anna Seghers während ihrer Zeit als Präsidentin des Schriftstellerverbands der DDR shinter den Kulissen ' gewirkt? Hier liegen die Aufgaben einer Seghers-Biographie, die natülich nur mit Hilfe von Archiven entsprechender Institutionen und privaten Nachlässen erarbeitet werden kann. Die vorliegende Einführung hält sich in der Hauptsache an das schriftstellerische Werk. Die Eckdaten der Biographie sind in einer knappen Zeittafel zusammengefaßt und werden bei der Werkanalyse vorausgesetzt. Die Werke sollen möglichst aus sich selbst heraus verstanden werden und nicht nach dem Erklärungsmodell einer kommunistischen und schon gar nicht einer antikommunistischen Ideologie. Es geht um den Platz von Anna Seghers in der Literatur- und Geistesgeschichte dieser Epoche, nicht so sehr um ihren Platz in einer sozialen und politischen Bewegung. 